\title{
Radio Capacity Estimation for Millimeter Wave 5G Cellular Networks Using Narrow Beamwidth Antennas at the Base Stations
}

\author{
AlMuthanna Turki Nassar, ${ }^{1,2}$ Ahmed Iyanda Sulyman, ${ }^{2}$ and Abdulhameed Alsanie ${ }^{2}$ \\ ${ }^{1}$ MRN Planning and Design Department, Mobily (Etihad Etisalat), P.O. Box 9979, Riyadh 11423, Saudi Arabia \\ ${ }^{2}$ Department of Electrical Engineering, King Saud University, P.O. Box 800, Riyadh 11421, Saudi Arabia
}

Correspondence should be addressed to AlMuthanna Turki Nassar; m.nassar@student.ksu.edu.sa

Received 11 March 2015; Accepted 16 June 2015

Academic Editor: Atsushi Mase

Copyright (C) 2015 AlMuthanna Turki Nassar et al. This is an open access article distributed under the Creative Commons Attribution License, which permits unrestricted use, distribution, and reproduction in any medium, provided the original work is properly cited.

\begin{abstract}
This paper presents radio frequency (RF) capacity estimation for millimeter wave (mm-wave) based fifth-generation (5G) cellular networks using field-level simulations. It is shown that, by reducing antenna beamwidth from $65^{\circ}$ to $30^{\circ}$, we can enhance the capacity of mm-wave cellular networks roughly by 3.0 times at a distance of $220 \mathrm{~m}$ from the base station (BS). This enhancement is far much higher than the corresponding enhancement of 1.2 times observed for $900 \mathrm{MHz}$ and $2.6 \mathrm{GHz}$ microwave networks at the same distance from the BS. Thus the use of narrow beamwidth transmitting antennas has more pronounced benefits in mm-wave networks. Deployment trials performed on an LTE TDD site operating on $2.6 \mathrm{GHz}$ show that 6 -sector site with $27^{\circ}$ antenna beamwidth enhances the quality of service (QoS) roughly by $40 \%$ and more than doubles the overall BS throughput (while enhancing the per sector throughput 1.1 times on average) compared to a 3-sector site using $65^{\circ}$ antenna beamwidth. This agrees well with our capacity simulations. Since mm-wave $5 \mathrm{G}$ networks will use arbitrary number of beams, with beamwidth much less than $30^{\circ}$, the capacity enhancement expected in $5 \mathrm{G}$ system when using narrow beamwidth antennas would be much more than three times observed in our simulations.
\end{abstract}

\section{Introduction}

Radio capacity is of great concern in cellular systems as it directly dictates the achievable data rates. The widespread use of smart phones and tablets and the availability of social network websites, where users can watch, download/share videos, and run many other mobile applications, led to an unprecedented increase in demand for high data rates. Global mobile data traffic reached 1.5 exabytes per month at the end of 2013, with data traffic growth rate of 81 percent in 2013 compared to 2012 [1]. This high demand for cellular data services, especially in large urban markets, puts pressure on operators to keep seeking solutions for realizing higher capacity in their networks. However, with such an explosive growth in data traffic, the existing mobile infrastructures including the $4 \mathrm{G}$ systems will be highly congested in the coming few years. This necessitates a disruptive next-generation capacity enhancement solution. Many of the requirements of what is called Beyond 4G (B4G) and 5G to reach multigigabits per second (Gbps) wireless data rates were discussed in [2], where the design of B4G and key technology components were presented. In this work, we examine the capacity of future 5G cellular networks on mm-wave frequency bands.

There is a growing belief that $\mathrm{mm}$-wave frequency bands $(30-300 \mathrm{GHz})$ will play a significant role in B4G and 5G cellular systems because of the huge bandwidths available for cellular services in that band $[3,4]$. Recent research works have thus devoted significant efforts to characterizing the $\mathrm{mm}$-wave channels. Path loss and propagation measurements were recently conducted for $\mathrm{mm}$-wave band at various frequencies: $28 \mathrm{GHz}, 38 \mathrm{GHz}, 60 \mathrm{GHz}$, and $72 \mathrm{GHz}$. The work in [5] presented a variety of indoor and outdoor propagation measurements where it was suggested that $28 \mathrm{GHz}$ and $38 \mathrm{GHz}$ bands can in fact be used for cellular systems when 
employing steerable directional antennas at the base station (BS) and mobile station (MS). It was also shown in [6] that, with adaptive antenna array beamforming, multi-Gbps data rates can be supported for mm-wave mobile cellular deployments at $28 \mathrm{GHz}$. Recent studies in [7-9] have also shown that adequate outdoor coverage is possible up to $220 \mathrm{~m}$ approximately for $\mathrm{mm}$-wave channels. Such cell size allows the use of low power microcell or picocell base stations with highly directional antennas in conjunction with multibeams techniques for more improvements. The work in [8] presented the achievable radio frequency (RF) coverage and system capacity using mm-wave based cellular systems based on field-level simulations, while the work in [9] develops mmwave path loss model based on real-world measurements at 28 and $38 \mathrm{GHz}$. It is shown in $[8,9]$ that, based on realistic BS radius of $220 \mathrm{~m}$, about four times the number of existing cell sites may be required in the mm-wave based network. In [10], theoretical analysis on the probability of coverage for $\mathrm{mm}$-wave cellular networks was presented, while [11] derives statistical models for key channel parameters including the path loss, number of spatial clusters, angular dispersion, and outage, based on real-world measurements at 28 and $73 \mathrm{GHz}$ in New York City. In this paper, we employ field-level network simulations, typically used by mobile radio operators for cellular planning, to estimate the field-level capacity performance of mm-wave cellular systems at $28 \mathrm{GHz}$ for various antenna beamwidths. We show that narrow beamwidth antennas have more pronounced capacity enhancements in $\mathrm{mm}$-wave networks than in their microwave counterparts.

\section{Radio Capacity Expression for Microwave and mm-Wave $5 \mathrm{G}$ Networks}

An MS located or moving in the coverage area of a serving BS will receive useful signals from the serving BS and interfering signals from other cochannel BS's. The server signal strength varies even when the MS is not moving due to the largescale fading and small-scale fading factors and also due to the interference received from neighboring cochannel BS's. All these factors determine the strength of the carrier-tointerference ratio (CIR) received by the MS. The CIR is the quotient between the average received modulated carrier power and the average received cochannel interfering power. A large CIR results in high quality of service (QoS), while a small CIR results in low QoS. Radio capacity is dependent on $\mathrm{CIR}$ and the available bandwidth $(B)$. The radio capacity for any wireless system can be estimated using the expression

$$
C=B \log _{2}(1+\mathrm{CIR})
$$

where $B$ denotes the RF channel bandwidth of the system and CIR denotes the carrier-to-interference ratio at various distances from the serving BS [9]. The CIR, in decibels (dB), can be estimated as

$$
\begin{aligned}
\mathrm{CIR}_{\mathrm{dB}} & =10 \log _{10}(\mathrm{CIR}) \\
& =10 \log _{10}\left(\frac{P_{\text {rec }}(\text { Serving BS })}{\sum P_{\text {rec }}(\text { Interfering BS's })}\right),
\end{aligned}
$$

where $P_{\text {rec }}$ is the received signal strength in watts, either from the serving BS or from the interfering BS's at any distance.

The received signal strength at various distances " $d$ " from the BS, $P_{\text {rec }}(d)$, can be estimated as

$$
P_{\mathrm{rec}}(d)[\mathrm{dB}]=P_{t}[\mathrm{~dB}]-\mathrm{PL}(d)[\mathrm{dB}],
$$

where $P_{t}[\mathrm{~dB}]$ denotes the transmitter (TX) power in $\mathrm{dB}$ and $\operatorname{PL}(d)[\mathrm{dB}]$ is the path loss at distance " $d$," also in dB. PL $(d)[d B]$ can be estimated using the Hata/COST231Hata models for microwave networks operating below $2 \mathrm{GHz}$ or using the Stanford University Interim (SUI) model for microwave networks above $2 \mathrm{GHz}$ [9]. For the mm-wave $5 \mathrm{G}$ networks, $\mathrm{PL}(d)[\mathrm{dB}]$ can be estimated using the modified SUI model presented in [9] for the $28 \mathrm{GHz}$ and $38 \mathrm{GHz}$ bands.

\subsection{Capacity Estimation for Microwave and $m m$-Wave Net-} works Using Narrow Beamwidth BS Antennas. In this section we estimate the radio capacity for $\mathrm{mm}$-wave cellular networks in comparison with its microwave counterparts when reducing the transmitting antenna beamwidth from $65^{\circ}$ to $30^{\circ}$. We used trisector BS with TX height of $20 \mathrm{~m}$ and EIRP of $63.5 \mathrm{dBm}$ ( $P t=43 \mathrm{dBm}$, TX antenna gain is $20.5 \mathrm{dBi}$ for $30^{\circ}$ beamwidth and $17 \mathrm{dBi}$ for $65^{\circ}$ beamwidth). A benchmark of $P_{\text {rec }}(d) \geq-75 \mathrm{dBm}$ outdoor is used at various locations $d$ within the cell for estimating coverage distance. For the $\mathrm{mm}$ wave network, we used this benchmark to arrive at a coverage distance of $220 \mathrm{~m}$, as reported in [5, 9].

Figure 1 shows the RF coverage pattern for a serving BS (site-58) in a $28 \mathrm{GHz} \mathrm{mm}$-wave network for both $65^{\circ}$ and $30^{\circ}$ antenna beamwidth. The circles around the server BS indicate the tiers of interfering BS's; the inner-most circle denotes the first-neighbor sites, the next circle denotes the second-neighbor sites, and so forth. Figure 2 displays the corresponding RF patterns for the interfering neighbors, with significant received signal strength, on the sector of site-58 in the direction of the arrow shown in the figure. Using the TEMS cell planner [12] and the propagation PL models introduced in [9] for mm-waves at $28 \mathrm{GHz}$, we measured the received signals from the serving base station (site-58) and the interfering neighbors while moving away from the serving site-58 (upward) in steps of $10 \mathrm{~m}$ up to a distance of $220 \mathrm{~m}$. Note that site ID's are on the right-hand side of each site in these figures. Measurement samples are shown in Tables 1 and 2 , respectively, for the $65^{\circ}$ and $30^{\circ}$ antenna beamwidth.

It can be seen from Tables 1 and 2 that deploying narrow beamwidth antennas in mm-wave based $5 \mathrm{G}$ networks results in less interference. The received signals from all neighboring sites are less in Table 2 compared to Table 1 especially for the neighboring sites $46,48,56,67$, and 69 . We can see that for these neighboring sites we did not receive any detectable signal at certain transmitter-receiver (TX-RX) separation distances for the case of $30^{\circ}$ beamwidth as was received for the case of $65^{\circ}$ beamwidth.

Using the measurement samples in Tables 1 and 2 and applying (1) and (2), we estimate the radio capacity for the $\mathrm{mm}$-wave based $5 \mathrm{G}$ cellular network operating on $28 \mathrm{GHz}$ and compare the results with microwave counterparts operating on $900 \mathrm{MHz}$ and $2.6 \mathrm{GHz}$, using both $65^{\circ}$ and $30^{\circ}$ 

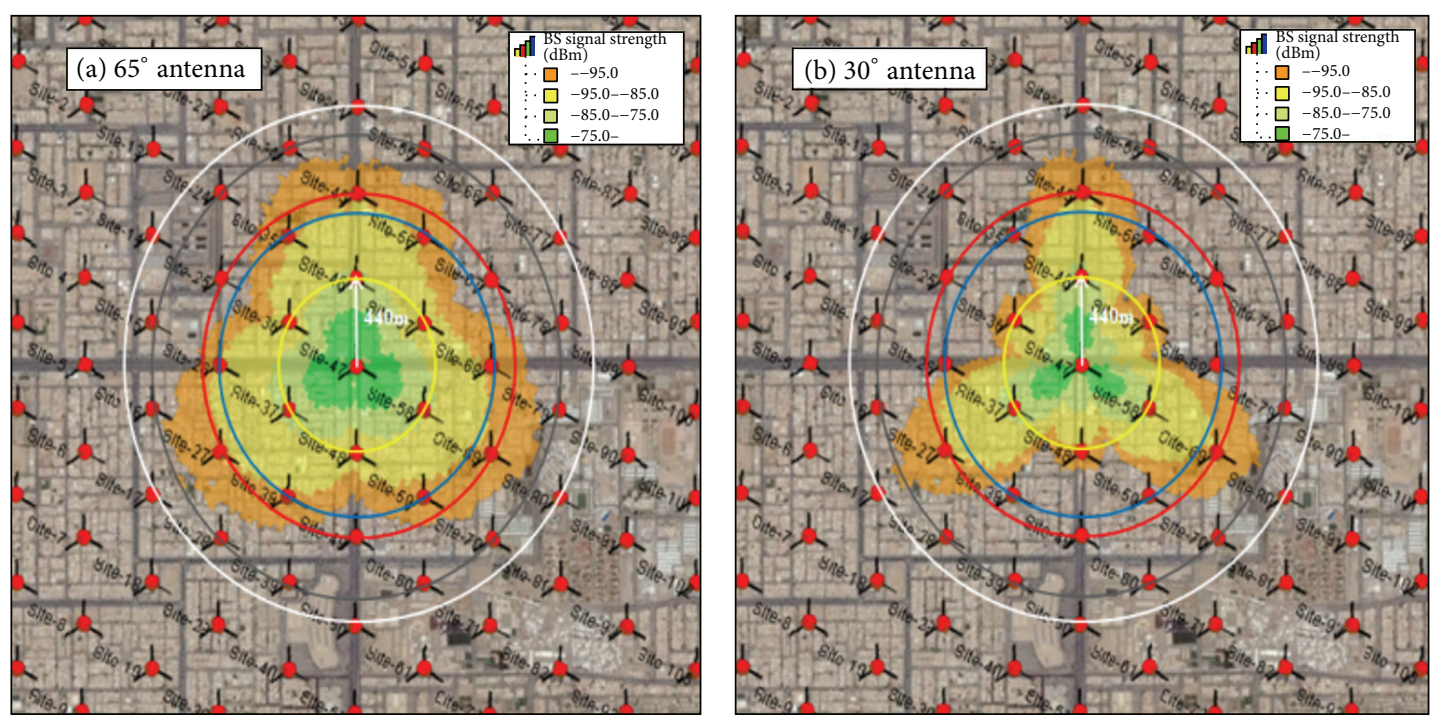

FIGURE 1: RF coverage of serving trisector BS (site-58) for the sector in the direction of the arrow for $f_{c}=28 \mathrm{GHz}$ and various TX antenna beamwidths: (a) $65^{\circ}$, (b) $30^{\circ}$.
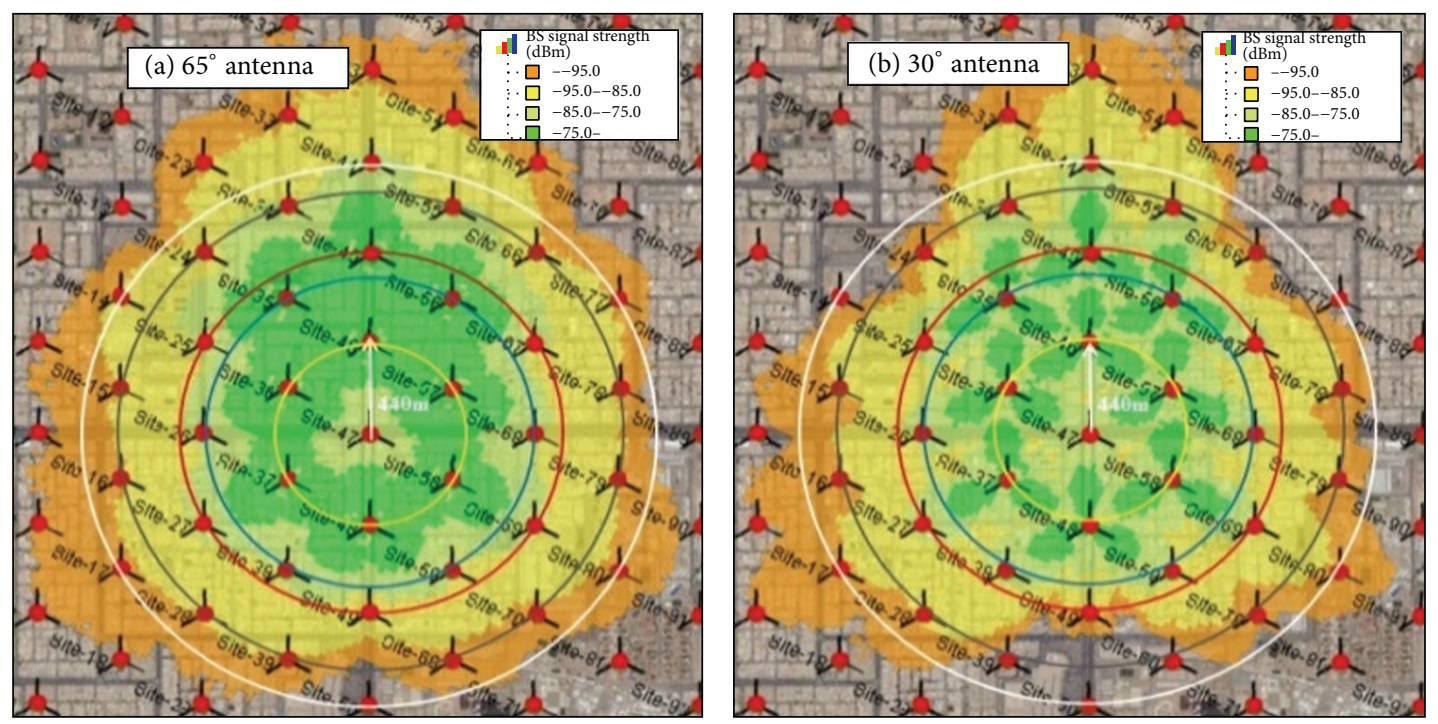

FIGURE 2: RF coverage of interfering trisector BS's with significant received signal strength on the sector of site-58 in the direction of the arrow for $f_{c}=28 \mathrm{GHz}$ and various TX antenna beamwidths: (a) $65^{\circ}$, (b) $30^{\circ}$.

antenna beamwidths. The resulting capacity enhancements for mm-wave network operating on $28 \mathrm{GHz}$ when reducing antenna beamwidth from $65^{\circ}$ to $30^{\circ}$ are shown in Figure 3, while counterparts results for microwave networks operating on $900 \mathrm{MHz}$ and $2.6 \mathrm{GHz}$ are shown in Figures 4 and 5, respectively. In general, from Figures 3, 4, and 5, it can be observed that for both the microwave and the $\mathrm{mm}$ wave networks the capacity decreases as the MS moves away from the serving BS, as expected. This is because of the decrease in the CIR as the MS moves away from the serving BS. In such case, the received signal from the serving BS becomes lower due to increasing path loss while the interference becomes higher. Also, it can be observed generally that, for both microwave and $\mathrm{mm}$-wave systems, the capacity is enhanced using narrower beamwidth transmitting antennas compared to the capacity when using the wider beamwidth antennas. The capacity of mm-wave networks however has much more pronounced enhancements using narrower beamwidth antenna than the microwave counterparts; and that is because the interference caused by an $\mathrm{mm}$-wave BS to MS's in the neighboring cells is way less than what is experienced in the microwave-based systems, thanks to the higher PL in the mm-wave bands which limits propagation distance away from the BS and provides better cell-edge coverage than the microwave-based networks $[8,9]$.

This is an important new insight. For example, the result in Figure 3 suggests that the capacity of $28 \mathrm{GHz} \mathrm{mm}$-wave 
TABLE 1: Carrier and interference measurements for the $28 \mathrm{GHz}$ mm-wave networks using $65^{\circ}$ beamwidth TX antennas for the trisector BS's.

\begin{tabular}{lccccccccccc}
\hline \multirow{2}{*}{ Site ID } & \multirow{2}{*}{ Server/neighbor } & \multicolumn{9}{c}{ Received signals in dBm at various TX-RX separation distances (m) } \\
& & $d=20$ & $d=40$ & $d=60$ & $d=80$ & $d=100$ & $d=120$ & $d=150$ & $d=170$ & $d=190$ & $d=220$ \\
\hline 58 & Server & -63.03 & -64.25 & -64.88 & -65.9 & -66.77 & -67.52 & -68.84 & -70.32 & -72.58 & -75.19 \\
57 & 1st neighbor & -90.25 & -88.41 & -87.51 & -86.54 & -85.56 & -83.67 & -80.63 & -79.9 & -78.8 & -75.95 \\
68 & 1st neighbor & -82.61 & -82.33 & -81.16 & -80.2 & -79.59 & -80.15 & -80.83 & -81.31 & -82.9 & -83.34 \\
69 & 1st neighbor & -90.48 & -90.67 & -90.67 & -90.07 & -91.25 & -90.59 & -89.98 & -91.27 & -91 & -92.23 \\
59 & 1st neighbor & -84.62 & -85.48 & -86.37 & -87.2 & -87.92 & -87.93 & -91.08 & -91.77 & -92.44 & -92.89 \\
48 & 1st neighbor & -94.7 & -94.44 & -94.44 & -94.97 & -96 & -95.65 & -95.66 & -96.17 & -96.17 & -95.85 \\
47 & 1st neighbor & -83.67 & -83.76 & -82.36 & -82.94 & -83.21 & -83.55 & -83.03 & -93.48 & -93.48 & -84.31 \\
46 & 2nd neighbor & -97.09 & -96.42 & -96.17 & -95.3 & -95.3 & -94.55 & -93.01 & -92.82 & -92.82 & -91.93 \\
67 & 2nd neighbor & - & - & - & - & - & - & - & - & - & -99.85 \\
56 & 3rd neighbor & -96.17 & -95.2 & -95.2 & -94.56 & -93.51 & -91.02 & -90.68 & -89.9 & -89.08 & -88.81 \\
\hline
\end{tabular}

TABLE 2: Carrier and interference measurements for $28 \mathrm{GHz}$ mm-wave networks using $30^{\circ}$ beamwidth TX antennas for the trisector BS’s.

\begin{tabular}{lccccccccccc}
\hline \multirow{2}{*}{ Site ID } & \multirow{2}{*}{ Server/neighbor } & \multicolumn{9}{c}{ Received signals in $\mathrm{dBm}$ at various TX-RX separation distances (m) } \\
& & $d=20$ & $d=40$ & $d=60$ & $d=80$ & $d=100$ & $d=120$ & $d=150$ & $d=170$ & $d=220$ & $d=250$ \\
\hline 58 & Server & -58.65 & -60.38 & -60.86 & -62.18 & -63.2 & -64.17 & -66.24 & -68.86 & -73.47 & -75.13 \\
57 & 1st neighbor & -95.8 & -94.08 & -93.5 & -92.17 & -90.59 & -87.41 & -86.46 & -83.47 & -81.4 & -79.15 \\
68 & 1st neighbor & -83.53 & -81.72 & -80.72 & -81.16 & -81.86 & -82.98 & -84.45 & -88.63 & -92.65 & -96.3 \\
69 & 1st neighbor & -96.86 & -97.7 & -97.82 & -98.29 & -99.79 & -99.79 & - & - & - & - \\
59 & 1st neighbor & -84.77 & -85.29 & -86.21 & -87.08 & -87.53 & -87.76 & -90.66 & -91.35 & -91.98 & -92.62 \\
48 & 1st neighbor & -99.42 & - & - & - & - & - & - & - & - & - \\
47 & 1st neighbor & -84.28 & -82.78 & -83.22 & -84.33 & -85.53 & -87.25 & -89.01 & -91.53 & -96.52 & -98.94 \\
46 & 2nd neighbor & - & - & - & - & -98.77 & -97.71 & -95.9 & -94.79 & -90.26 & -90.26 \\
67 & 2nd neighbor & - & - & - & - & - & - & - & - & - & - \\
56 & 3rd neighbor & - & - & - & - & -99.25 & -96.74 & -94.99 & -94.01 & -91.6 & -90.39 \\
\hline
\end{tabular}

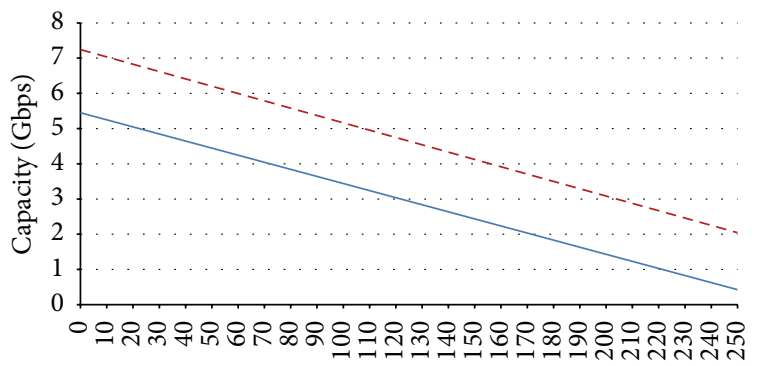

TX-RX separation distance $(\mathrm{m})$

- - mm-wave network $\left(f_{c}=28 \mathrm{GHz}, B=1\right.$ $\mathrm{GHz}, 30$-degree antenna)

mm-wave network ( $f_{c}=28 \mathrm{GHz}, B=1$ $\mathrm{GHz}, 65$-degree antenna)

Figure 3: Capacity trend in Gbps for $28 \mathrm{GHz}$ mm-wave networks using $65^{\circ}$ and $30^{\circ}$ transmitting antenna beamwidths.

cellular networks is roughly enhanced by 3.0 times at a distance of $220 \mathrm{~m}$ from the BS, when reducing TX antenna beamwidth from $65^{\circ}$ to $30^{\circ}$. This enhancement is far much higher than the corresponding enhancement of 1.2 times observed in Figures 4 and 5 for the $900 \mathrm{MHz}$ and $2.6 \mathrm{GHz}$ microwave networks at the same distance from the BS. This result means that a capacity enhancement of $300 \%$ at celledge is possible in a $28 \mathrm{GHz} \mathrm{mm}$-wave network by just reducing antenna beamwidth from $65^{\circ}$ to $30^{\circ}$. Mm-wave $5 \mathrm{G}$

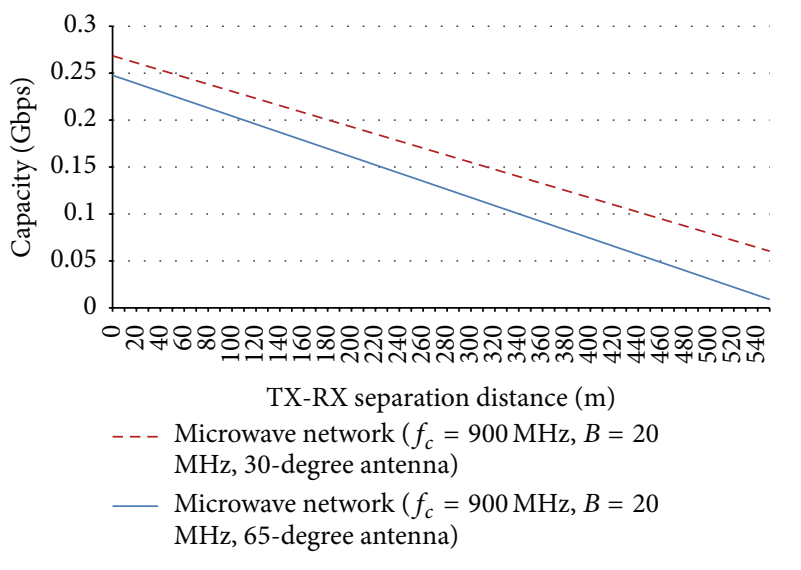

FIGURE 4: Capacity trend in Gbps for $900 \mathrm{MHz}$ microwave networks using $65^{\circ}$ and $30^{\circ}$ transmitting antenna beamwidths.

networks will employ multibeam RF signals, with arbitrary beamwidth much less than $30^{\circ}$. Therefore it is expected that this narrowing of antenna beamwidth transmission strategy alone can scale up capacity significantly and help realize gigabits per second data rates everywhere in the $5 \mathrm{G}$ network, including cell edges. For indoor coverage, cell enhancers and in-building RF infrastructures may be needed to insure providing acceptable received power levels inside buildings with satisfying in-building data rates. 


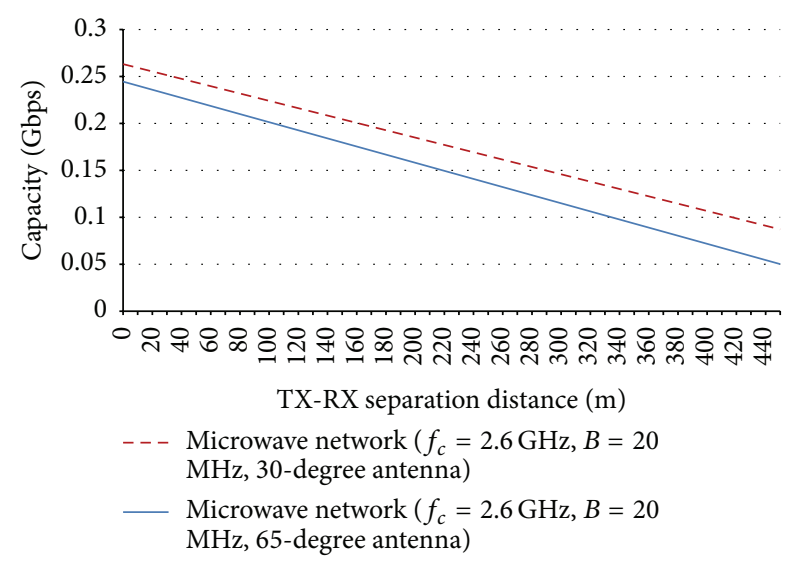

FIGURE 5: Capacity trend in Gbps for $2.6 \mathrm{GHz}$ microwave networks using $65^{\circ}$ and $30^{\circ}$ transmitting antenna beamwidths.

\section{Field Deployment Trials for Narrow Beamwidth Antennas Using 4G LTE}

Next we present results from field deployment trials on the capacity enhancements achieved when migrating from $65^{\circ}$ to $27^{\circ}$ antenna beamwidth in a $4 \mathrm{G}$ LTE network using multibeam transmitting antennas. This trial is performed on an LTE TDD site which operates at $2.6 \mathrm{GHz}$ band. The site is originally a 3 -sector site with antenna height of $30 \mathrm{~m}$. It was then converted to a 6-sector site during the trials using the following configurations. Each $4 \mathrm{Tx}-4 \mathrm{Rx}$ sector is split into two $2 \mathrm{Tx}-2 \mathrm{Rx}$ sectors. Therefore, for a 3 -sector $4 \mathrm{Tx}-4 \mathrm{Rx}$ site, this solution will result in a 6 -sector $2 \mathrm{Tx}-2 \mathrm{Rx}$ site using 4 -port multibeam transmitting antenna, with each of 2 ports used for one $2 \mathrm{Tx}-2 \mathrm{Rx}$ sector. The new 4-port multibeam antenna has a gain of $20.1 \mathrm{dBi}$, horizontal beamwidth of $27^{\circ}$, and vertical beamwidth of $4.8^{\circ}$, while the old 4 -port antenna has a gain of $17.3 \mathrm{dBi}$ and horizontal and vertical beamwidths of $65^{\circ}$ and $6.5^{\circ}$, respectively. Large numbers of users are on this LTE test site before the trial since it serves a dense urban area in Dammam city, Saudi Arabia.

The BS full (overall) throughput is the throughput when all the radio blocks ( $\mathrm{RB}$ ) at the $\mathrm{BS}$ sites are loaded up. The RB is the basic unit of radio resource in LTE. One RB occupies $180 \mathrm{KHz}$ portion of the $20 \mathrm{MHz}$ carrier signal bandwidth used. The BS full throughput reports are as shown in Figures 6 and 7, respectively, for the downlink (DL) and uplink (UL). As shown in these figures, significant enhancements in the UL and DL full throughput are observed using 6 sectors. The DL BS full throughput is improved by $112 \%$, while the UL BS full throughput is improved by $19 \%$. The DL per sector throughput corresponds to our capacity simulation above since we consider only one sector (transmission from the BS sector to the users), in the capacity simulations. The average per sector DL throughput is enhanced only 1.1 times using 6-sector site compared to the 3-sector counterpart for the $2.6 \mathrm{GHz}$ microwave network. This agrees closely with the 1.2 capacity enhancements reported in our simulations. Although deployment trails for mm-wave network are currently not possible, the close agreement between the $2.6 \mathrm{GHz}$

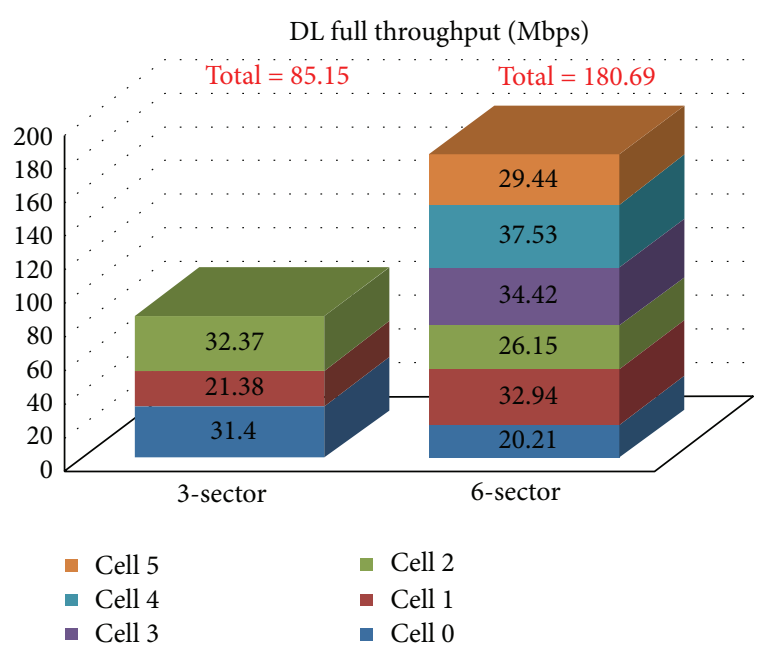

Figure 6: The cell full downlink throughput in Mbps before and after the 6-sector integration.

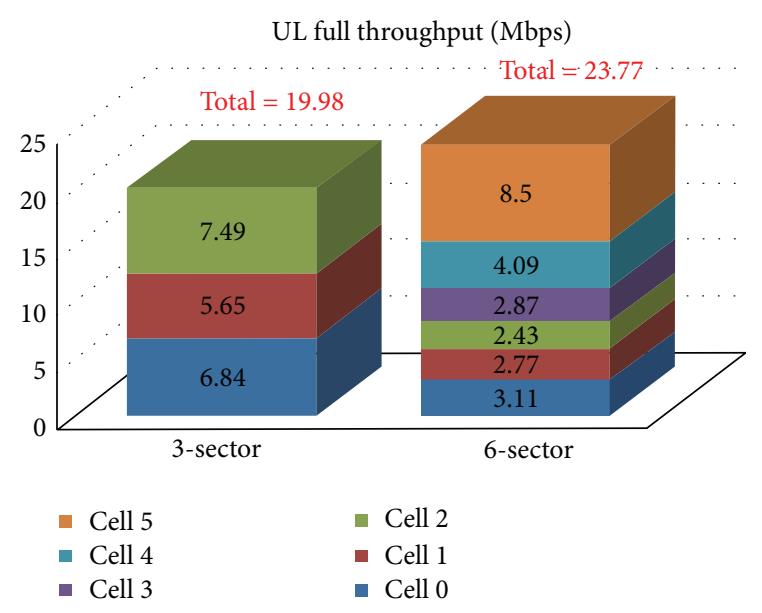

FIGURE 7: The cell full uplink throughput in Mbps before and after the 6-sector integration.

microwave network simulation and the field trials results is another way to validate our simulation results obtained for the mm-wave case.

For the user throughput, significant enhancements are observed after the 6-sector integration as shown in Figure 8. $\mathrm{DL}$ average user throughput is more than doubled in some cases, while the UL average user throughput improved only slightly. This is due to the fact that directional antennas are currently not implemented on the user mobile devices participating in this trial. Thus the beamwidth changes at the BS will only affect DL transmission to users appreciably. If narrow beamwidth antennas are introduced at the BS and mobiles, then both UL and DL enhancements would be observed. This case cannot be implemented in the deployment trials yet.

The service drop rate is the rate at which connections are lost during the service. This is a measure of the QoS provided by the operator. The service drop rate significantly improved after the integration of 6 sectors as shown in Figure 9. It is enhanced by $42 \%$ on average compared to the 3 -sector scenario. 


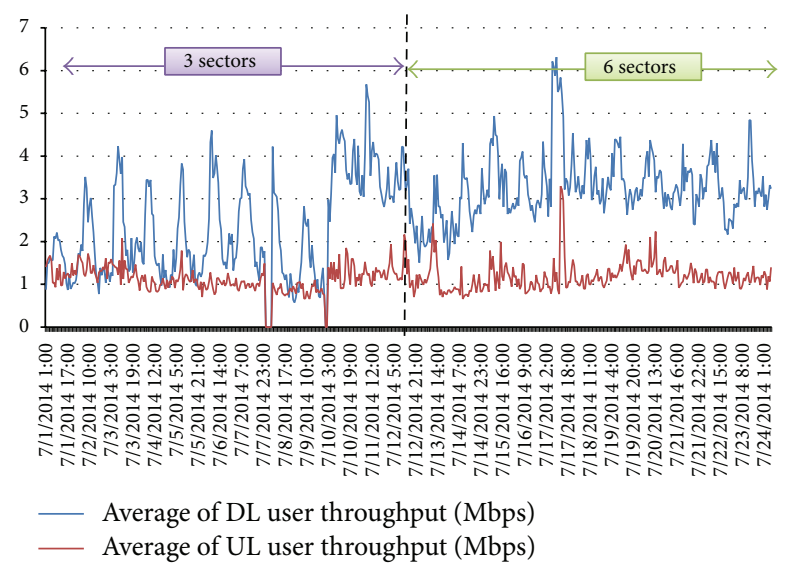

FIGURE 8: The uplink and downlink user throughput in Mbps before and after the 6-sector integration.

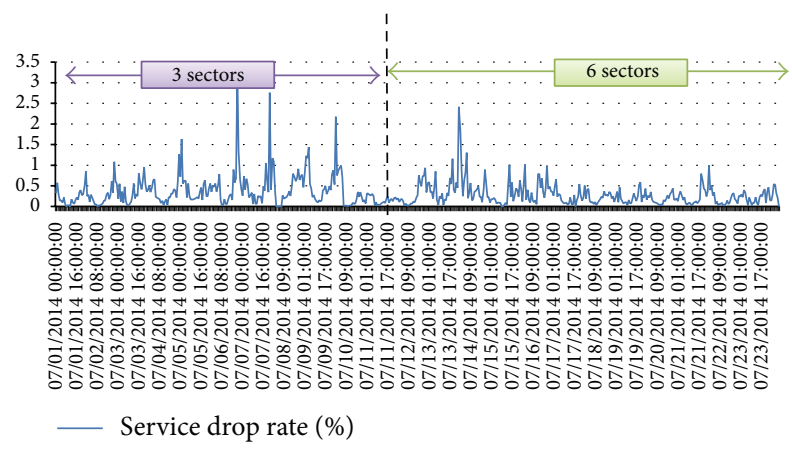

FIGURE 9: The site service drop rate before and after the 6-sector integration.

The site trial results presented above corroborate the trends observed in our simulations; namely, capacity and QoS can be significantly enhanced migrating from $65^{\circ}$ antenna beamwidth to narrower beamwidths. 5G networks will employ narrow antenna beamwidth similar to the ones used in this trial; therefore we expect this solution to enhance the capacity and QoS in 5G networks significantly.

\section{Conclusions}

This paper presents radio capacity estimation for mm-wave based 5G cellular networks using field-level simulations. It is shown that the capacity of microwave and mm-wave networks can be enhanced using narrow beamwidth transmitting antennas. More pronounced capacity enhancements, roughly more than double, are observed for the mm-wave networks compared to the microwave counterpart at a TXRX separation distance of $220 \mathrm{~m}$, when moving from $65^{\circ}$ to $30^{\circ} \mathrm{TX}$ antennas. Thus it is concluded that narrow beamwidth solution can help 5G systems to realize gigabits per second data rates everywhere in the network including the cell edges. Deployment trials performed on an LTE TDD site corroborate our findings in the simulation studies. The trial results show that BS overall DL throughput will be doubled and the service drop rate will be enhanced roughly by $40 \%$.

\section{Conflict of Interests}

The authors declare that there is no conflict of interests regarding the publication of this paper.

\section{Acknowledgment}

This work was funded by the National Plan for Science, Technology and Innovation (MAARIFAH), King Abdulaziz City for Science and Technology, Saudi Arabia, Award Number 11ELE1854-02.

\section{References}

[1] Cisco, "Cisco visual networking index: global mobile data traffic forecast update, 2013-2018," White Paper, 2015, https://www .cisco.com/c/en/us/solutions/collateral/service-provider/visualnetworking-index-vni/white_paper_c11-520862.pdf.

[2] P. E. Mogensen, K. Pajukoski, B. Raaf et al., "B4G local area: high level requirements and system design," in Proceedings of the IEEE Globecom Workshops, pp. 613-617, December 2012.

[3] F. Boccardi, R. W. Heath Jr., A. Lozano, T. L. Marzetta, and P. Popovski, "Five disruptive technology directions for 5G," IEEE Communications Magazine, vol. 52, no. 2, pp. 74-80, 2014.

[4] Z. Pi and F. Khan, "An introduction to millimeter-wave mobile broadband systems," IEEE Communications Magazine, vol. 49, no. 6, pp. 101-107, 2011.

[5] T. S. Rappaport, S. Sun, R. Mayzus et al., "Millimeter wave mobile communications for 5G cellular: it will work!," IEEE Access, vol. 1, pp. 335-349, 2013.

[6] S. Rajagopal, S. Abu-Surra, Z. Pi, and F. Khan, "Antenna array design for multi-Gbps mmwave mobile broadband communication," in Proceedings of the IEEE Global Telecommunications Conference (GLOBECOM '11), pp. 1-6, Houston, Tex, USA, December 2011.

[7] S. Rangan, T. S. Rappaport, and E. Erkip, "Millimeter-wave cellular wireless networks: potentials and challenges," Proceedings of the IEEE, vol. 102, no. 3, pp. 366-385, 2014.

[8] A. T. Nassar, A. I. Sulyman, and A. Alsanie, "Achievable RF coverage and system capacity using millimeter wave cellular technologies in 5G networks," in Proceedings of the 27th IEEE Canadian Conference on Electrical and Computer Engineering (CCECE '14), pp. 1-6, IEEE, Toronto, Canada, May 2014.

[9] A. I. Sulyman, A. T. Nassar, M. K. Samimi, G. R. MacCartney Jr., T. S. Rappaport, and A. Alsanie, "Radio propagation path loss models for $5 \mathrm{G}$ cellular networks in the 28 and $38 \mathrm{GHz}$ millimeter wave bands," IEEE Communications Magazine, vol. 52, no. 9, pp. 78-86, 2014.

[10] S. Akoum, O. El Ayach, and R. W. Heath, "Coverage and capacity in mmWave cellular systems," in Proceedings of the Conference Record of the Forty Sixth Asilomar Conference on Signals, Systems and Computers (ASILOMAR '12), pp. 688-692, IEEE, Pacific Grove, Calif, USA, November 2012.

[11] M. R. Akdeniz, Y. Liu, M. K. Samimi et al., "Millimeter wave channel modeling and cellular capacity evaluation," IEEE Journal on Selected Areas in Communications, vol. 32, no. 6, pp. 1164-1179, 2014.

[12] TEMS CellPlanner (TCP) tool, 2014, http://www.mentum.com. 

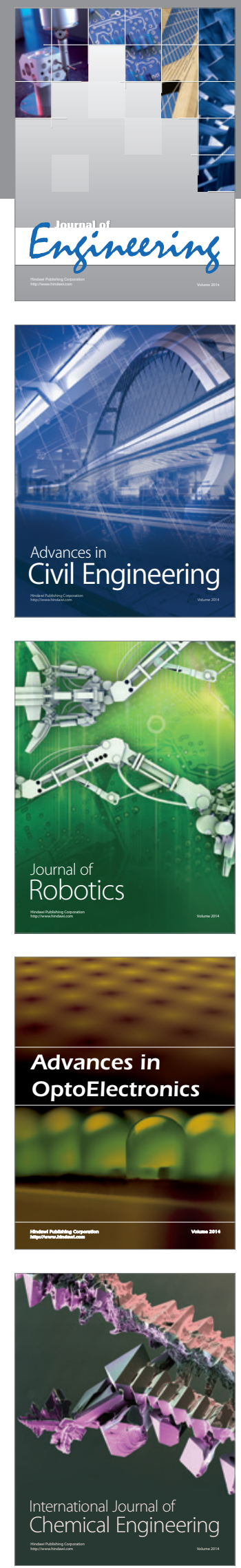

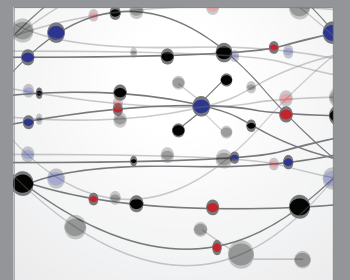

The Scientific World Journal
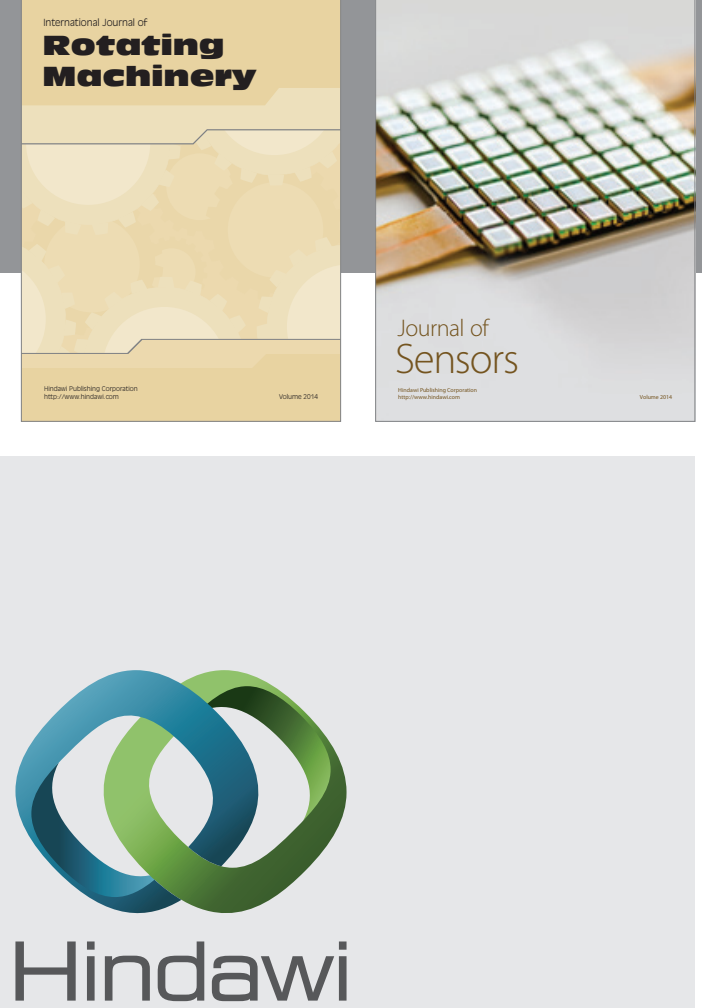

Submit your manuscripts at http://www.hindawi.com
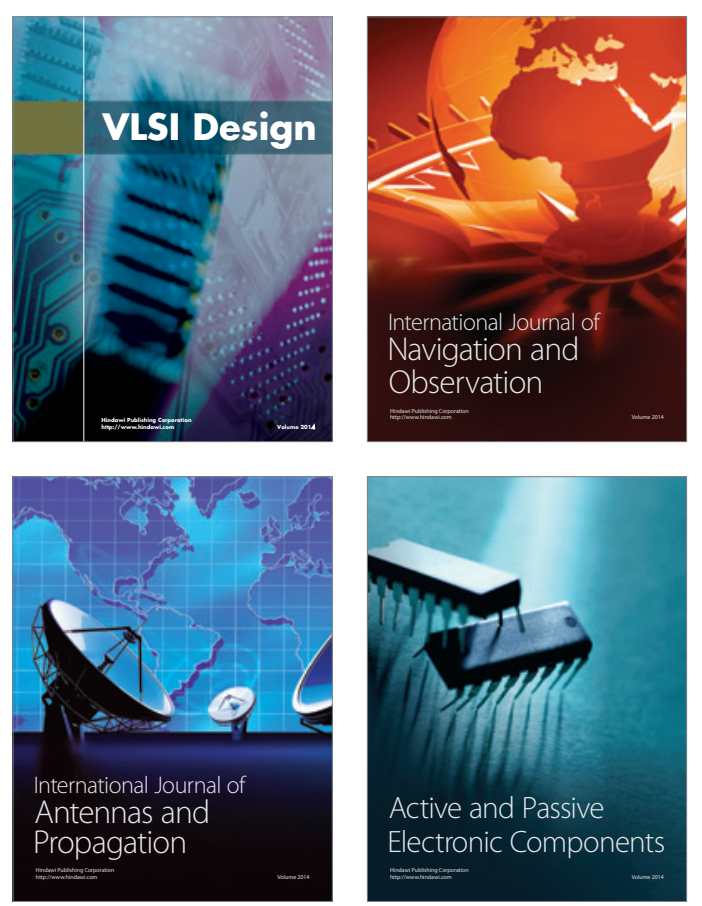
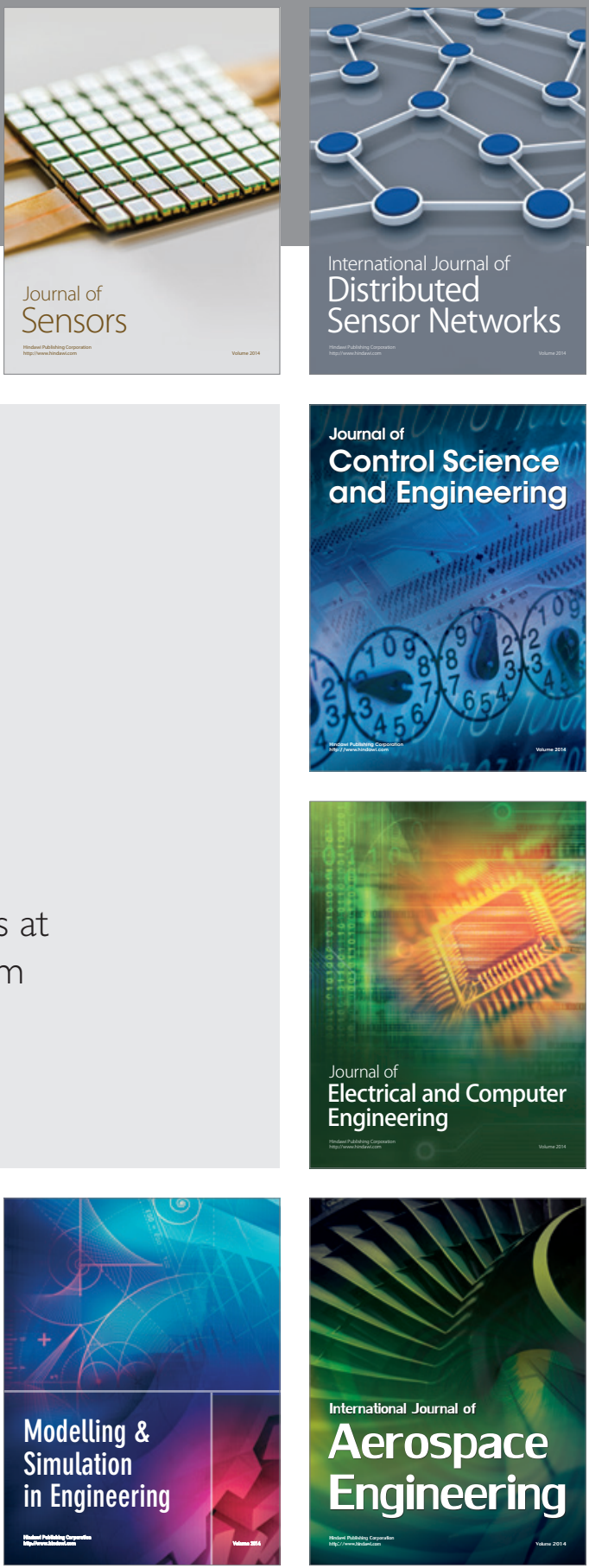

Journal of

Control Science

and Engineering
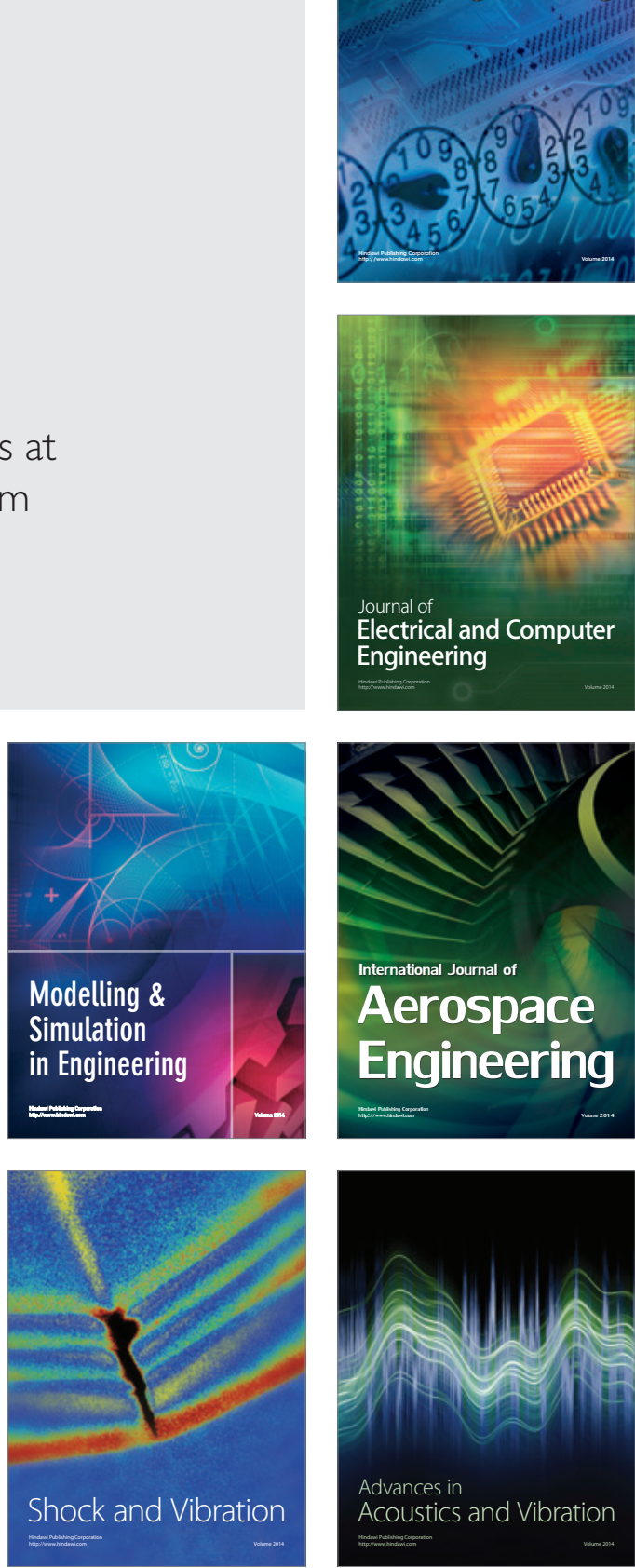